\title{
Simultaneous pancreas-kidney transplantation: The role in the treatment of type 1 diabetes and end-stage renal disease
}

\author{
Alex T. Jiang, BHSc; ${ }^{*}$ Neal Rowe, MD, FRCSC; ${ }^{\dagger}$ Alp Sener, MD, PhD, FRCSC,; Patrick Luke, MD, FRCSC ${ }^{\S}$ \\ *Department of Surgery, Division of Urology; Schulich School of Medicine and Dentistry, Western University, London, ON; †'Department of Surgery, Division of Urology, Western University; Multi-Organ \\ Transplant Program, London Health Sciences Center, London, ON; §Department of Surgery, Division of Urology, Western University; Multi-Organ Transplant Program; Matthew Mailing Centre for Translational \\ Transplant Studies, London Health Sciences Centre; Department of Microbiology and Immunology; Centre for Human Immunology; Western University, London, ON
}

Cite as: Can Urol Assoc J 2014;8(3-4):135-8. http://dx.doi.org/10.5489/cuaj.1597

Published online April 14, 2014.

\section{Abstract}

Type 1 diabetes mellitus (DM) is one of the most common and debilitating diseases to affect the world. Many patients are afflicted by microvascular and macrovascular complications, and succumb to end-stage renal disease (ESRD). Although dialysis and insulin therapy provides better glycemic control, it nonetheless significantly decreases a patient's quality of life. Moreover, they cannot reverse ESRD or alleviate complications. Simultaneous pancreas-kidney (SPK) transplantation has revolutionized the way we manage type $1 \mathrm{DM}$; it provides a physiological means of achieving normoglycemia while rendering patients free of dialysis. Understanding this procedure is important because it is becoming a more common management strategy for patients with type $1 \mathrm{DM}$. In this review, we will begin with a brief summary of type 1 DM, followed by a comprehensive description of SPK procedure, including the history and technique. We will then present the outcomes of transplantation.

\section{Introduction}

Type 1 diabetes mellitus (DM) is one of the most common chronic diseases of childhood caused by insulin deficiency secondary to autoimmune destruction of pancreatic betacells. The condition affects about 1.4 million individuals in United States, with an annual incidence of 17 cases per 100000 children. ${ }^{1}$ Unmanaged, it can lead to severe longterm complications. These include microvascular events, such as retinopathy, neuropathy and nephropathy, and macrovascular diseases involving cerebrovascular, coronary or peripheral vascular systems., ${ }^{2,3}$ These complications are largely attributed to hyperglycemia resulting from poor insulin secretion. Consequently, the mortality rate for type 1 DM is high $-13 \%$ after 20 years of disease. ${ }^{4}$

One of the most signification complications of type 1 DM is end-stage renal disease (ESRD). ${ }^{5}$ It initially manifests as microalbuminuria with subsequent progression to pro- teinuria. Without intervention, $80 \%$ of these cases lead to nephropathy and ultimately, ESRD (glomerular filtration rate $<15 \mathrm{~mL} / \mathrm{min} / 1.73 \mathrm{~m}^{2}$ ). Eligible patients with ESRD require dialysis or renal transplantation for long-term management. ${ }^{6}$

The Diabetes Control and Complication Trial (DCCT) demonstrated that tight glycemic control, achieved through intensive insulin therapy, slows the progression and reduces the risk of developing micro- and macro-vascular complications. ${ }^{7}$ Despite use of insulin pumps and intensive insulin therapy, no exogenous delivery of insulin has been able to sustain normoglycemia as effectively as a functional pancreas. As such, allogeneic pancreas transplantation was developed to achieve normoglycemia and insulin independence. The combination of pancreas and kidney transplantation can render a patient free of both insulin and dialysis with prevention of further diabetic complications, and occasional reversal of established disease. ${ }^{8}$

\section{Pancreas transplantation history}

The first pancreas transplantation was performed in 1966 by William Kelly and Richard Lillehei at the University of Minnesota in conjunction with a kidney transplant to treat a diabetic uremic patient. ${ }^{9}$ Early procedures were associated with significant morbidity and mortality and performed in low numbers in very select patients. With the advent of cyclosporine and improvements in surgical techniques, 1 -year graft survival rates exceeded $70 \%$ in 1980 s. ${ }^{10}$ To date, more than 32000 cases have been performed worldwide with ever improving outcomes. ${ }^{11}$

Currently, there are 3 methods of solid organ pancreas transplantation. Most (83\%) procedures are performed in the context of simultaneous pancreas-kidney (SPK) transplantation where the pancreas is transplanted at the same time as the kidney. The second method is pancreas after kidney (PAK) transplantation (12\%) where a pancreas is transplanted to a patient who previously received kidney transplantation. The third method is pancreas transplant alone (PTA) 
(5\%), which involves transplantation of a solitary pancreas to a diabetic patient with normal renal function. This is performed to counteract life-threatening hypoglycemic unawareness or rapidly progressive diabetic complications refractory to intensive insulin therapy. ${ }^{12}$

\section{Selection process}

The SPK procedure is usually reserved for patients with type $1 \mathrm{DM}$ as confirmed by low or absent level of C-peptide. ${ }^{13}$ Candidates may also have significant nephropathy or ESRD, along with complications, such as hypoglycemic unawareness, recurrent hospitalization from diabetic ketoacidosis, progressive retinopathy, enteropathy and neuropathy (Table 1). ${ }^{14}$

\section{Surgical procedure}

The techniques used for SPK transplantation are diverse and institution-dependent. Most transplant centres use the intraperitoneal approach for graft placement. The pancreas is transplanted to a heterotopic location, usually the right iliac fossa, while the kidney is transplanted to the contralateral iliac fossa. This approach results in fewer peripancreatic fluid collections and wound complications. An alternative approach involves extraperitoneal and ipsilateral placement of both grafts. ${ }^{15}$

Arterial anastomosis may be performed by conjoining the donor superior mesenteric artery and splenic artery to a $Y$ graft of the recipient external or common iliac artery. The donor portal vein is anastomosed to the external iliac vein if systemic drainage is provided. An alternative approach is anastomosis of donor portal vein to superior mesenteric vein if portal venous drainage is available. ${ }^{16}$ Although this was performed to reduce lipid dysregulation and rejection rates, contemporary studies have shown very little differences in overall long-term outcomes between systemic and portal drainages. ${ }^{17}$

\section{Outcomes}

\section{Survival}

It is believed that the SPK procedure prolongs patient survival beyond the survival advantage associated with renal transplantation alone. The 5- and 10-year patient survival rates for SPK transplantation is $87 \%$ and $70 \%$, respectively. ${ }^{18}$ This is significantly better than the survival rates for patients with type 1 DM receiving maintenance dialysis and who are on transplant waiting list. ${ }^{19}$ However, due to inherent biases in listing candidates for transplants and the differences in donor age between SPK and solitary kidney (SK) transplant cohorts, the true survival benefit conferred by the pancreas

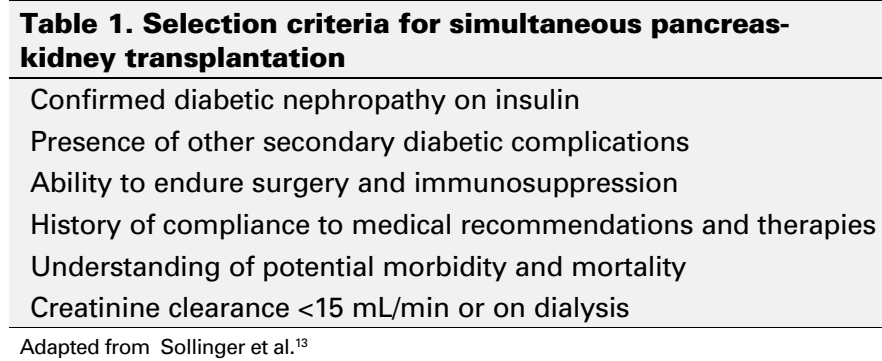

is unknown.

Graft survival rates are excellent. The pancreatic allograft survival rate is $86 \%$ at 1 year and $53 \%$ at 10 years, while kidney survival rate is $>95 \%$ at 1 year and $60 \%$ at 10 years. ${ }^{18,20}$ The lower 1 -year graft survival rates for the pancreas are secondary to early transplant complications, including thrombosis, pancreatic fistula, and infection. ${ }^{18,20}$

\section{Quality of life}

Pancreas transplantation can improve quality of life by eliminating diabetes-associated complications, including hypo/ hyperglycemia, metabolic derangements, insulin dependence, glucose monitoring and dietary restrictions. ${ }^{21}$ Smith and colleagues compared pre- and post-transplant quality of life and found significant improvement following SPK transplantation. ${ }^{22}$ Snort-form-36 Mental Component Summary scores were significantly higher 2 years post-transplant compared to pre-transplant (51.8 vs. 46.8). Similar results were obtained from the Physical Component Summary (PCS) score (48.1 vs. 40.6). ${ }^{22}$

\section{Glycemic control}

The vast majority of patients achieve complete insulin independence over the short and long term following solid-organ pancreas transplant. In fact, glycemic control is far superior to that achieved by insulin pump or islet-cell transplants. ${ }^{23}$ Mora and colleagues demonstrated that recipients achieved long-term normoglycemic state following SPK transplant. ${ }^{24}$ During the 15-year follow-up, HbA1c level remained within the normal range with no significant difference between the first and the last year of follow-up $(4.68 \%$ vs. $4.76 \%$, $p>0.05) .{ }^{24}$ Fasting glucose level also remained stable during the same period ( 3.94 vs. $4.38 \mathrm{mmoL} / \mathrm{L}, p>0.05) .^{24}$ However, oral glucose tolerance test (OGTT) demonstrated decreased pancreatic response, indicating certain deterioration in the functional capability of the allograft over the long term. ${ }^{24}$ It is unknown whether this is the effect of immunosuppressive medications (i.e., tacrolimus, sirolimus and prednisone) on islet-cell function, insulin resistance, immune-related chronic changes, or a combination thereof. 


\section{Vascular}

The pancreas transplant does not reverse established macrovascular disease in recipients. Instead, it is believed to slow down the progression of disease in this high-risk population. Nevertheless, 5 years after transplantation, the prevalence of cerebrovascular disease (CVD), coronary heart disease (CHD) and peripheral vascular disease (PVD) is still 33\%, $41 \%$ and $41 \%$, respectively. Ten years after transplantations, the risk increased only slightly to $41 \%, 50 \%$ and 50 $\%$, respectively. ${ }^{25}$ Using peripheral thermography studies, it is believed that microvascular perfusion is improved postpancreas transplant as a result of better glycemic control. ${ }^{26}$

\section{Neuropathy}

Previous studies demonstrated a benefit of SPK transplant for diabetic polyneuropathy. Kennedy and colleagues analyzed the effect of pancreas transplantation on peripheral motor, sensory and autonomic nerve function based on indexes of nerve conduction velocity and muscle action potential. ${ }^{27}$ After 12 months of follow-up, they found a significant improvement in motor and sensory indices. This is supported by Martinenghi and colleagues who found that a sustained normoglycemic state can improve nerve function even if polyneuropathy is advanced. ${ }^{28}$

\section{Nephropathy}

Most patients with type $1 \mathrm{DM}$ and ESRD receive SPK to improve their renal function. Fioretto and colleagues reported that 10 years of sustained normoglycemia post-transplant reversed features of diabetic nephropathy. ${ }^{29}$ It significantly improved glomerular and tubular lesions, and reduced the thickness of glomerular basement membrane and mesangial matrix. A decrease in urinary albumin excretion rate was also observed (20 mg/day vs. $103 \mathrm{mg} /$ day) highlighting improvement in renal function. However, improvements in diabetic nephropathy post-transplant need to be balanced with nephrotoxicity incurred by the use of immunosuppressive agents, such as tacrolimus and cyclosporine. ${ }^{30}$

\section{Retinopathy}

Diabetic retinopathy (DR) is the most common microvascular complication of diabetes. Several studies have reported conflicting results about the effects of SPK on retinopathy. However, most recent studies indicate that SPK, with subsequent normalization of blood glucose level, can improve or normalize retinal lesions. ${ }^{31}$ Following SPK transplantation, $14 \%$ of non-blind eyes showed improvement, $76 \%$ remained stable and only $10 \%$ progressed further. ${ }^{32} \mathrm{~A}$ separate study reported an improvement in post-transplant visual acuity in
$32 \%$ of the eyes and frequency/severity of vitreous hemorrhages in $46 \%$ of eyes. ${ }^{31}$ It may take up to 4 years before noticeable functional improvement in retinopathy and acuity may be observed. Pancreas transplantation, however, cannot reverse established visual loss.

\section{Conclusion}

SPK transplantation is the most effective treatment for patients with type $1 \mathrm{DM}$ and ESRD. It addresses renal failure and provides physiological means of attaining stable insulin secretion. Although it involves major surgery and is not without risks, SPK transplantation nonetheless increases patient survival, enhances quality of life and prevents progression of diabetic complications. As such, SPK transplantation should be considered in all eligible patients.

Acknowledgement: This work was supported in part by a grant from the Urology Care Foundation Research Scholars Program and the Frank and Marion Hinman Urology Research Fund.

Competing interests: Alex Jiang, Dr. Rowe, Dr. Sener and Dr. Luke all declare no competing financial or personal interests.

This paper has been peer-reviewed.

\section{References}

1. van der Boog P, Ringers J, Paul LC, et al. Simultaneous kidney-pancreas transplantation: The preferred option for patients with type I diabetes mellitus and approaching end-stage renal disease. Transplant Rev 2004;18:129-38. htrp://dx.doi.org/10.1016/j.trre.2004.04.003

2. Lipshutz GS, Wilkinson AH. Pancreas-kidney and pancreas transplantation for the treatment of diabetes mellitus. Endocrinol Metab Clin North Am 2007;36:1015-38. http://dx.doi.org/10.1016/i. ecl.2007.07.010

3. Rayhill SC, D'Alessandro AM, Odorico IS, et al. Simultaneous pancreas-kidney transplantation and living related donor renal transplantation in patients with diabetes: Is there a difference in survival? Ann Surg 2000;23:417-23. http://dx.doi.org/10.1097/00000658-200003000-00015

4. Stadler $M$, Auinger $M$, Anderwald $C$, et al. Long-term mortality and incidence of renal dialysis and transplantation in type 1 diabetes mellitus. J Clin Endocrinol Metab 2006;91:3814-20. http://dx.doi. org/10.1210/ic.2006-1058

5. Gross JL, de Azevedo MJ, Silveiro SP, et al. Diabetic nephropathy: Diagnosis, prevention, and treatment. Diabetes Care 2005;28:164-76. http://dx.doi.org/10.2337/diacare.28.1.164

6. Nishimura R, Dorman JS, Bosnyak Z, et al. Incidence of ESRD and survival after renal replacement therapy in patients with type 1 diabetes: A report from the Allegheny County Registry. Am I Kidney Dis 2003;42:117-24. http://dx.doi.org/10.1016/S0272-6386(03)00414-1

7. The Diabetes Control and Complications Trial Research Group. The effect of intensive treatment of diabetes on the development and progression of long-term complications in insulin-dependent diabetes mellitus. $N$ Engl J Med 1993;329: 977-86. http://dx.doi.org/10.1056/NEJM199309303291401

8. Lerner, SM. Kidney and pancreas transplantation in type 1 diabetes mellitus. Mount Sinai Journal of Medicine 2008;75:372-84. http://dx.doi.org/10.1002/msi.20056

9. Kelly WD, Lillehei RC, Merkel FK, et al. Allotransplantation of the pancreas and duodenum along with the kidney in diabetic nephropathy. Surgery 1967;61:827-37.

10. Stratta RJ, Shokouh-Amiri MH, Egidi MF, et al. Long-term experience with simultaneous kidney-pancreas transplantation with portal-enteric drainage and tacrolimus/mycophenolate mofetil-based immunosuppression. Clin Transplant 2003;17:69-77. http://dx.doi.org/10.1034/i.1399-0012.17.s9.13.x 
Jiang et al.

11. Perosa M, Boggi U, Cantarovich D, et al. Pancreas transplantation outside the USA: an update. Curr Opin Organ Transplant 2011;16:135-41. http://dx.doi.org/10.1097/MOT.0b013e3283424ccc

12. Ming $C S$, Chen ZH. Progress in pancreas transplantation and combined pancreas-kidney transplantation. Hepatobiliary Pancreat Dis Int 2007;6:17-23.

13. Sollinger HW, Odorico IS, Knechtle SJ, et al. Experience with 500 simultaneous pancreas-kidney transplants. Ann Surg 1998;228:284-96. http://dx.doi.org/10.1097/00000658-199809000-00002

14. Freise CE, Narumi S, Stock PG, et al. Simultaneous pancreas-kidney transplantation: An overview of indications, complications, and outcomes. West J Med 1999;170:11-8.

15. Becker BN, Odorico JS, Becker YT, et al. Simultaneous pancreas-kidney and pancreas transplantation. J Am Soc Nephrol 2001;12:2517-27.

16. Gaber A0, Shokouh-Amiri MH, Grewel HP, et al. A technique for portal pancreas transplantation with enteric drainage. Surg Gynecol Obstet 1993;177:417-9.

17. Stratta RJ, Shokouh-Amiri MH, Egidi MF, et al. A prospective comparison of simultaneous kidney-pancreas transplantation with systemic-enteric versusportal-enteric drainage. Ann Surg 2001;233:740-51. http:// dx.doi.org/10.1097/00000658-200106000-00003

18. McCullough KP, Keith DS, Meyer KH, et al. Kidney and pancreas transplantation in the United States, 1998-2007: Access for patients with diabetes and end-stage renal disease. Am J Transplant 2009;9:894906. http://dx.doi.org/10.1111/i.1600-6143.2009.02566.x

19. Meier-Kriesche $\mathrm{HU}, \mathrm{Ojo} \mathrm{AO}$, Port FK, et al. Survival improvement among patients with end-stage renal disease: Trends over time for transplant recipients and wait-listed patients. J Am Soc Nephrol 2001;12:1293-6.

20. Ziaja J, Chudek J, Kolonko A, et al. Does simultaneously transplanted pancreas improve long-erm outcome of kidney transplantation in type 1 diabetic recipients? Transplant Proc 2011;43:3097-101. http:// dx.doi.org/10.1016/j.transproceed.2011.08.020

21. Isla PP, Moncho VJ, Torras RA, et al. Quality of life in simultaneous pancreas-kidney transplant recipients. Clin Transplant 2009;23:600-5. http://dx.doi.org/10.1111/i.1399-0012.2009.01054.x

22. Smith GC, Trauer T, Kerr PG, et al. Prospective quality-of-life monitoring of simultaneous pancreas and kidney transplant recipients using the 36-Item Short Form Health Survey. Am J Kidney Dis 2010;55:698707. http://dx.doi.org/10.1053/i.ajkd.2009.12.025
23. Gerber PA, Paulicek V, Demartines N, et al. Simultaneous islet-kidney vs pancreas-kidney transplantation in type 1 diabetes mellitus: A 5 year single centre follow-up. Diabetologia 2008;51:110-9. http:// dx.doi.org/10.1007/s00125-007-0860-4

24. Mora M, Ricart MJ, Casamitjana R, et al. Pancreas and kidney transplantation: Long-term endocrine function. Clin Transplant 2010;24:236-40. http://dx.doi.org/10.1111/j.1399-0012.2010.01261.x

25. Biesenbach G, Konigsrainer A, Gross C, et al. Progression of macrovascular diseases is reduced in type 1 diabetic patients after more than 5 years successful combined pancreas-kidney transplantation in comparison to kidney transplantation alone. Transplant Int 2005; 18:1054-60. http://dx.doi.org/10.1111/i.14322277.2005.00182.x

26. Larsen JL, Colling CW, Ratanasuwan T, et al. Pancreas transplantation improves vascular disease in patients with type 1 diabetes. Diabetes Care 2004;27:1706-11. http://dx.doi.org/10.2337/diacare.27.7.1706

27. Kennedy WR, Navarro $X$, Goetz $F$, et al. Effects of pancreatic transplantation on diabetic neuropathy. $N$ Engl J Med 1990;332:1031-7. http://dx.doi.org/10.1056/NEJM199004123221503

28. Martinenghi $S$, Comi $G$, Galardi $G$, et al. Amelioration of nerve conduction velocity following simultaneous kidney/pancreas transplantation is due to the glycaemic control provided by the pancreas. Diabetologia 1997;40:1110-2. http://dx.doi.org/10.1007/s001250050795

29. Fioretto $P$, Steffes MW, Sutherland DER, et al. Reversal of lesions of diabetic nephropathy after pancreas transplantation. N Engl J Med 1998;339:69-75. http://dx.doi.org/10.1056/NEJM199807093390202

30. Fioretto P, Steffes MW, Mihatsch MJ, et al. Cyclosporine associated lesions in native kidneys of diabetic pancreas transplant recipients. Kidney Int 1995;48:489-95. http://dx.doi.org/10.1038/ki.1995.318

31. Pearce IA, llango B, Sells RA, et al. Stabilization of diabetic retinopathy following simultaneous pancreas and kidney transplant. Br J Ophthalmol 2000;84:736-40. http://dx.doi.org/10.1136/bjo.84.7.736

32. Chow VC, Pai RP, Chapman JR, et al. Diabetic retinopathy after combined kidney-pancreas transplantation. Clin Transplantation 1999;13:356-62. http://dx.doi.org/10.1034/j.1399-0012.1999.130413.x

Correspondence: Dr. Patrick Luke, Professor, Department of Surgery, Division of Urology, Western University, University Hospital, Room C4-207, 339 Windermere Rd, London, ON, N6A 5A5; fax: 519-663-3858; patrick.luke@|hsc.on.ca patrick.luke@lhsc.on.co 